\title{
Enantioselective Synthesis of 10-epi-Anamarine via an Iterative Dihydroxylation Sequence
}

\author{
Dong Gao and George A. O’Doherty* \\ Department of Chemistry, West Virginia University \\ Morgantown, WV 26506
}

\section{Supporting Information:}

General Methods and Materials. ${ }^{1} \mathrm{H}$ and ${ }^{13} \mathrm{C}$ NMR spectra were recorded on Jeol (270 $\mathrm{MHz})$ and Varian VXR-600 (600 MHz) spectrometers. Chemical shifts are reported relative to internal tetramethylsilane $(\delta 0.00 \mathrm{ppm})$ or $\mathrm{CDCl}_{3}(\delta 7.26 \mathrm{ppm})$ for ${ }^{1} \mathrm{H} \mathrm{NMR}$ and $\mathrm{CDCl}_{3}(\delta 77.0 \mathrm{ppm})$ for ${ }^{13} \mathrm{C} \mathrm{NMR}$. Infrared (IR) spectra were obtained on a Prospect MIDAC FT-IR spectrometer. Optical rotations were measured with a Jasco DIP-370 digital polarimeter in the solvent specified. Melting points were determined with Electrothermal Mel-Temp apparatus and are uncorrected. Flash column chromatography was performed on ICN reagent 60 (60-200 mesh) silica gel. Analytical thin-layer chromatography was performed with precoated glass-backed plates (Whatman K6F 60A,, $\mathrm{F}_{254}$ ) and visualized by quenching of fluorescence and by charring after treatment with $p$ anisaldehyde or phosphomolybdic acid or potassium permanganate stain. $R_{f}$ values are obtained by elution in the stated solvent ratios (v/v). Ether, THF, methylene chloride and triethylamine were dried by passing through activated alumina ( 8 x 14 mesh) column with argon gas pressure. Commercial reagents were used without purification unless otherwise noted. Melting points are uncorrected. Air and/or moisture-sensitive reactions were carried out under an atmosphere of argon/nitrogen using oven/flamed-dried glassware and standard syringe/septa techniques. 
(E,4'S,5'S)-ethyl 3-(2',2',5'-trimethyl-1',3'-dioxolan-4'-yl)acrylate (10).

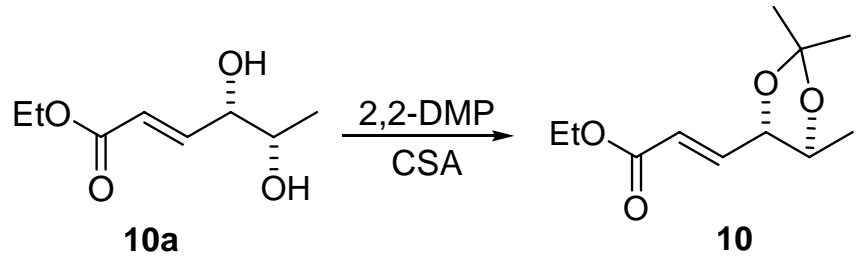

To a stirred solution of (E,4S,5S)-ethyl 4,5-dihydroxyhex-2-enoate 10a (300 mg, 1.72 $\mathrm{mmol})$ in $2 \mathrm{~mL}$ of $\mathrm{CH}_{2} \mathrm{Cl}_{2}$ at room temperature was added 2,2-DMP $(0.42 \mathrm{ml}, 3.44 \mathrm{mmol})$ and CSA ( $8 \mathrm{mg}, 2 \mathrm{~mol} \%$ ). The reaction was stirred for $3 \mathrm{~h}$ and quenched with saturated aqueous sodium bicarbonate $(10 \mathrm{~mL})$ and the aqueous layer was extracted with ether $(3 \mathrm{x}$ $15 \mathrm{~mL})$. The combined organic layers were washed with brine $(25 \mathrm{~mL})$, and dried over anhydrous sodium sulfate. After removal of the solvents in vacuo, flash chromatography on silica gel (9:1 (v/v) hexanes/EtOAc) afforded (E)-ethyl 3-((4S,5S)-2,2,5-trimethyl-1,3dioxolan-4-yl)acrylate 10 as a viscous oil $(310 \mathrm{mg}, 83 \%): R_{f}=0.56(7: 3(\mathrm{v} / \mathrm{v})$ hexane/EtOAc); $[\alpha]^{25}+6.5^{\circ}$ (c 2.2, $\mathrm{CH}_{2} \mathrm{Cl}_{2}$ ); IR (thin film, $\mathrm{cm}^{-1}$ ) 2985, 2936, 2876, 1723, $1663,1454,1373,1302,1249,1175,1107,1036,980 ;{ }^{1} \mathrm{H}$ NMR $\left(\mathrm{CDCl}_{3}, 600 \mathrm{MHz}\right) \delta$ $6.85(\mathrm{dd}, J=15.6,6 \mathrm{~Hz}, 1 \mathrm{H}), 6.11(\mathrm{dd}, J=15.6,1.2 \mathrm{~Hz}, 1 \mathrm{H}), 4.22(\mathrm{q}, J=7.2 \mathrm{~Hz}, 1 \mathrm{H})$, 4.19 (q, $J=7.2 \mathrm{~Hz}, 1 \mathrm{H}), 4.07$ (qd, $J=6,2.4 \mathrm{~Hz}, 1 \mathrm{H}), 3.83$ (ddd, $J=8.4,6,2.4 \mathrm{~Hz}, 1 \mathrm{H}$ ), $1.31(\mathrm{~d}, J=6 \mathrm{~Hz}, 3 \mathrm{H}), 1.44$ (s, 3H), 1.41 (s, 3H), 1.29 (t, $J=7.2 \mathrm{~Hz}, 3 \mathrm{H}) ;{ }^{13} \mathrm{C}$ NMR $\left(\mathrm{CDCl}_{3}, 150 \mathrm{MHz}\right): \delta 165.9,143.4,122.8,119.2,81.6,76.4,60.6,27.2,26.6,16.6,14.2$; HRMS (CI) calcd for $\left[\mathrm{C}_{11} \mathrm{H}_{18} \mathrm{O}_{4}+\mathrm{Na}\right]^{+}: 237.1097$, Found: 237.0995.

(2S,3S)-ethyl

2,3-dihydroxy-3-((4'S,5'S)-2',2',5'-trimethyl-1',3'-dioxolan-4'yl)propanoate (11a):

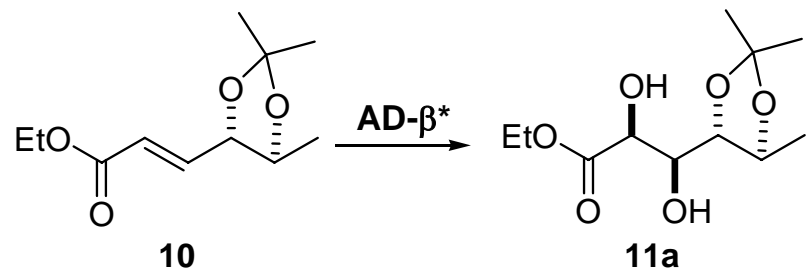

Into a $50 \mathrm{~mL}$ round bottom flask was added $2 \mathrm{~mL}$ of $t-\mathrm{BuOH}, 2 \mathrm{~mL}$ of water, $\mathrm{K}_{3} \mathrm{Fe}(\mathrm{CN})_{6}$ (461 mg, $1.4 \mathrm{mmol}), \mathrm{K}_{2} \mathrm{CO}_{3}\left(193 \mathrm{mg}, 1.4 \mathrm{mmol}\right.$ ), $\mathrm{NaHCO}_{3}(117 \mathrm{mg}, 1.4 \mathrm{mmol}$ ), $\mathrm{MeSO}_{2} \mathrm{NH}_{2}$ (45 mg, $0.47 \mathrm{mmol}$ ), (DHQD) 2 PHAL (15 mg, $0.02 \mathrm{mmol}, 4 \mathrm{~mol} \%$ ), and 
$\mathrm{OsO}_{4}(3 \mathrm{mg}, 0.01 \mathrm{mmol}, 2 \mathrm{~mol} \%)$. The mixture was stirred at room temperature for about 15 minutes and then cooled to $0{ }^{\circ} \mathrm{C}$. To this solution was added a solution (E)-ethyl 3-((4S,5S)-2,2,5-trimethyl-1,3-dioxolan-4-yl)acrylate 10 (100 mg, $0.47 \mathrm{mmol})$ in $1 \mathrm{~mL}$ of $\mathrm{CH}_{2} \mathrm{Cl}_{2}$ and the reaction was stirred vigorously at $0{ }^{\circ} \mathrm{C}$ for $12 \mathrm{~h}$. The reaction was quenched with solid sodium sulfite $(100 \mathrm{mg})$ at room temperature. Then the mixture was filtered through a pad of celite/florisil and eluted with $(2 \times 20 \mathrm{~mL})$ ethyl acetate. The combined organic layers were dried over anhydrous sodium sulfate and the solvent was removed in vacuo. The crude product was purified by flash chromatography on silica gel $(7: 3 \quad(\mathrm{v} / \mathrm{v})$ hexane/EtOAc) to yield (2S,3S)-ethyl 2,3-dihydroxy-3-((4S,5S)-2,2,5trimethyl-1,3-dioxolan-4-yl)propanoate 11a (110 mg, 10:1 dr, 95\% yield) as a viscous oil. The major isomer was separated by column chromatography. Major isomer: white crystalline solid; $\mathrm{mp} 86-87^{\circ} \mathrm{C} ; R_{f}=0.42\left(6: 4(\mathrm{v} / \mathrm{v})\right.$ hexane/EtOAc); $[\alpha]^{25}{ }_{\mathrm{D}}+11.4^{\circ}$ (c 2 , $\mathrm{CH}_{2} \mathrm{Cl}_{2}$ ); IR (thin film, $\mathrm{cm}^{-1}$ ) 3334, 2987, 2937, 1735, 1662, 1578, 1416, 1331, 1298, 1140, 988, 884; ${ }^{1} \mathrm{H} \mathrm{NMR}\left(\mathrm{CDCl}_{3}, 600 \mathrm{MHz}\right) \delta 4.44(\mathrm{dd}, J=4.2,1.8 \mathrm{~Hz}, 1 \mathrm{H}), 4.30(\mathrm{q}, J=$ $7.2 \mathrm{~Hz}, 2 \mathrm{H}), 4.13$ (dq, $J=7.8,6 \mathrm{~Hz}, 1 \mathrm{H}), 3.90$ (ddd, $J=10.2,9,1.8 \mathrm{~Hz}, 1 \mathrm{H}), 3.61$ (ddd, $J$ $=9,7.8,1.2 \mathrm{~Hz}, 1 \mathrm{H}), 3.14(\mathrm{~d}, J=4.2 \mathrm{~Hz}, 1 \mathrm{H}), 2.20(\mathrm{~d}, J=10.2 \mathrm{~Hz}, 1 \mathrm{H}), 1.42(\mathrm{~s}, 3 \mathrm{H})$,

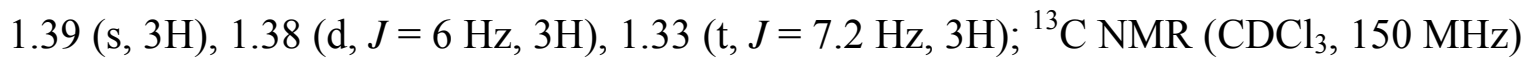
$\delta 173.3,108.6,80.9,76.5,73.7,70.7,62.3,27.4,26.9,19.4,14.1$; HRMS (CI) calcd for $\left[\mathrm{C}_{11} \mathrm{H}_{20} \mathrm{O}_{6}+\mathrm{Na}\right]^{+}:$271.1152, Found: 271.1163.

(2S,3R)-ethyl 2,2-dimethyl-5-((4R,5S)-2',2',5'-trimethyl-1',3'-dioxolan-4'-yl)-1,3dioxolane-4-carboxylate (11).

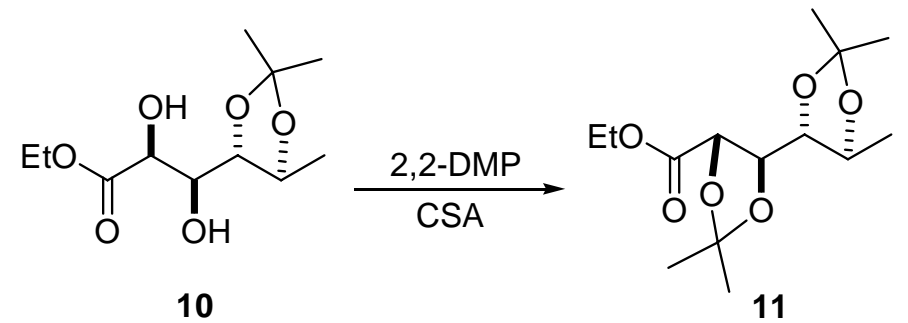

To a solution of diol $10(543 \mathrm{mg}, 2.19 \mathrm{mmol})$ in $5 \mathrm{~mL}$ of $\mathrm{CH}_{2} \mathrm{Cl}_{2}$ was added 2,2dimethoxypropane $(1.3 \mathrm{~mL}, 3.45 \mathrm{mmol})$ and CSA (20 mg, $4 \mathrm{~mol} \%)$ at room temperature. The reaction was stirred for $24 \mathrm{~h}$ and quenched with sat'd aqueous $\mathrm{NaHCO}_{3}$. The 
aqueous layer was extracted with $\mathrm{Et}_{2} \mathrm{O}$. The combined organic layers were wished with brine and dried over anhydrous $\mathrm{Na}_{2} \mathrm{SO}_{4}$, and concentrated to afford the crude product. Flash chromatography on silica gel $(9: 1(\mathrm{v} / \mathrm{v})$ hexane/EtOAc) provided compound 11 (504 mg, 80\% yield) as a colorless oil. $\mathrm{R}_{f}=0.61(7: 3(\mathrm{v} / \mathrm{v})$ hexane/EtOAc); IR (neat, $\left.\mathrm{cm}^{-1}\right)$ 2986, 1752; $[\alpha]_{\mathrm{D}}^{25}+19.8^{\circ}\left(\mathrm{c} 1, \mathrm{CHCl}_{3}\right) ;{ }^{1} \mathrm{H} \mathrm{NMR}\left(\mathrm{CDCl}_{3}, 600 \mathrm{MHz}\right): \delta 4.49$ (d, $J$ $=5.2,1 \mathrm{H}), 4.27(\mathrm{dd}, J=6.0,6.0 \mathrm{~Hz}, 1 \mathrm{H}), 4.23(\mathrm{q}, J=7.2 \mathrm{~Hz}, 1 \mathrm{H}), 4.22(\mathrm{q}, J=7.2 \mathrm{~Hz}$, 1H), 4.04 (dq, $J=7.8,6.0 \mathrm{~Hz}, 1 \mathrm{H}), 3.63$ (dd, $J=7.2,7.2 \mathrm{~Hz}, 1 \mathrm{H}), 1.44$ (s, 3H), 1.40 (s, $3 \mathrm{H}), 1.39$ (s, 3H), 1.34 (s, 3H), 1.33 (d, $J=6.0 \mathrm{~Hz}, 3 \mathrm{H}), 1.28$ (dd, $J=7.2,7.2 \mathrm{~Hz}, 3 \mathrm{H})$;

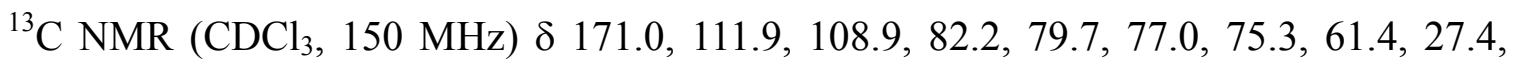
27.1, 26.7, 25.9, 18.4, 14.0; HRMS (CI) calcd for $\left[\mathrm{C}_{14} \mathrm{H}_{24} \mathrm{O}_{6}+\mathrm{Na}\right]^{+}: 311.1465$, Found: 311.1460 .

\section{((2S,3R)-2,2-dimethyl-5-((4'R,5'S)-2',2',5'-trimethyl-1',3'-dioxolan-4'-yl)-1,3-} dioxolane-4-yl)methanol (11a).

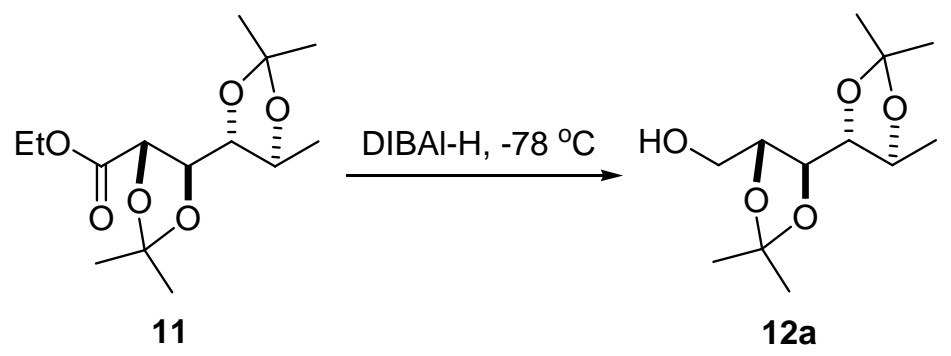

To a solution of ester $11(520 \mathrm{mg}, 0.52 \mathrm{mmol})$ in $8 \mathrm{~mL}$ of THF was added DIBAL-H (4.5 $\mathrm{ml}, 20 \% \mathrm{wt}$ in toluene, $5.4 \mathrm{mmol}$ ) dropwise at $-78{ }^{\circ} \mathrm{C}$. In $30 \mathrm{~min}$, the reaction was quenched by adding $1 \mathrm{~mL}$ of acetone and $5 \mathrm{~mL}$ of $10 \% \mathrm{NaOH}$ solution. The mixture was filtered through a pad of celite. The aqueous layer was extracted with $\mathrm{Et}_{2} \mathrm{O}$, the combined organic layers were wished with brine, dried over anhydrous $\mathrm{Na}_{2} \mathrm{SO}_{4}$ and concentrated to afford the crude product. Flash chromatography on silica gel $(7: 3(\mathrm{v} / \mathrm{v})$ hexane/EtOAc) provided compound 12a (411 mg, 93\% yield) as a colorless oil. $\mathrm{R}_{f}=0.20$ (7:3 (v/v) hexane/EtOAc); IR (neat, $\left.\mathrm{cm}^{-1}\right) 3369,2989,1456$; $[\alpha]^{25}-13.8$ (c 1, $\mathrm{CHCl}_{3}$ ); ${ }^{1} \mathrm{H}$ NMR $\left(\mathrm{CDCl}_{3}, 600 \mathrm{MHz}\right) \delta$ 4.09-4.03 (m, 1H), 3.80 (ddd, $\left.J=11.4,4.8,4.2 \mathrm{~Hz}, 1 \mathrm{H}\right)$, $3.76(\mathrm{dd}, J=8.4,7.8 \mathrm{~Hz}, 1 \mathrm{H}), 3.72(\mathrm{dd}, J=8.4,7.8 \mathrm{~Hz}, 1 \mathrm{H}), 3.50(\mathrm{dd}, J=8.4,7.8 \mathrm{~Hz}$, $1 \mathrm{H}), 2.40(\mathrm{dq}, J=4.8,4.2 \mathrm{~Hz}, 1 \mathrm{H}), 1.41(\mathrm{~s}, 3 \mathrm{H}), 1.40$ (s, 3H), 1.38 (s, 3H), 1.37 (d, $J=$ 
$6.0 \mathrm{~Hz}, 3 \mathrm{H}), 1.36(\mathrm{~s}, 3 \mathrm{H}) ;{ }^{13} \mathrm{C} \mathrm{NMR}\left(\mathrm{CDCl}_{3}, 150 \mathrm{MHz}\right) \delta 109.6,109.1,82.7,81.0,79.4$, 77.1, 62.7, 27.3, 26.9, 26.8, 26.7, 18.3; HRMS (CI) calcd for $\left[\mathrm{C}_{12} \mathrm{H}_{22} \mathrm{O}_{5}+\mathrm{Na}\right]^{+}: 269.1359$, Found: 269.1352 .

(2S,3R)-2,2-dimethyl-5-((4'R,5'S)-2',2',5'-trimethyl-1',3'-dioxolan-4'-yl)-1,3dioxolane-4-carbaldehyde (12).

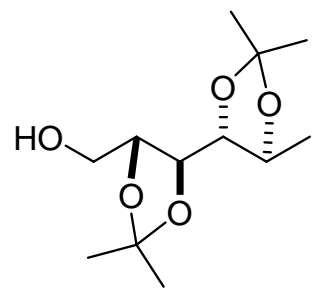

$12 a$

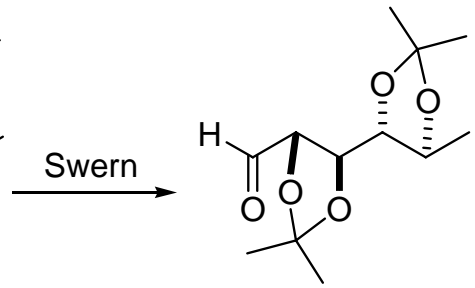

12

To a solution of oxalylchloride (174 mg, $1.37 \mathrm{mmol}$ ) in $3 \mathrm{~mL}$ of $\mathrm{CH}_{2} \mathrm{Cl}_{2}$ was added DMSO (134 mg, $1.71 \mathrm{mmol})$ at $-78{ }^{\circ} \mathrm{C}$. After stirring for $10 \mathrm{~min}$, alcohol 12a $(280 \mathrm{mg}$, $1.14 \mathrm{mmol}$ ) in $1 \mathrm{~mL}$ of $\mathrm{CH}_{2} \mathrm{Cl}_{2}$ was added dropwise. The mixture was stirred for another $10 \mathrm{~min}$, and then $\mathrm{Et}_{3} \mathrm{~N}(375 \mathrm{mg}, 3.3 \mathrm{mmol}$ ) was added. In $20 \mathrm{~min}$, the dry ice was removed and the solution was stirred for $30 \mathrm{~min}$ and quenched with sat'd aqueous $\mathrm{NaHCO}_{3}$. The aqueous layer was extracted with EtOAc. The combined organic layers were wished with brine, dried over anhydrous $\mathrm{Na}_{2} \mathrm{SO}_{4}$, and concentrated to afford the crude product. Flash chromatography on silica gel $(7: 3(\mathrm{v} / \mathrm{v})$ hexane/EtOAc) provided compound 12 (239 mg, 86\% yield) as a colorless oil. $\mathrm{R}_{f}=0.23$ (7:3 (v/v) hexane/EtOAc); IR $\left(\right.$ neat, $\left.\mathrm{cm}^{-1}\right)$ 2986, 1741; $[\alpha]_{\mathrm{D}}^{25}=-16.3^{\circ}\left(\mathrm{c} 1, \mathrm{CHCl}_{3}\right) ;{ }^{1} \mathrm{H}$ NMR $\left(\mathrm{CDCl}_{3}, 600 \mathrm{MHz}\right) \delta$ $9.76(\mathrm{~d}, J=0.6,1 \mathrm{H}), 4.48(\mathrm{dd}, J=6.0,1.2 \mathrm{~Hz}, 1 \mathrm{H}), 4.10(\mathrm{dd}, J=7.2,6.0 \mathrm{~Hz}, 1 \mathrm{H}), 4.04$ (ddd, $J=10.2,7.2,6.0 \mathrm{~Hz}, 1 \mathrm{H}), 3.64$ (dd, $J=7.8,7.2 \mathrm{~Hz}, 1 \mathrm{H}), 1.48$ (s, 3H), $1.42(\mathrm{~s}, 3 \mathrm{H}$ ), 1.39 (s, 3H), 1.37 (d, $J=6.0 \mathrm{~Hz}, 3 \mathrm{H}), 1.36(\mathrm{~s}, 3 \mathrm{H}) ;{ }^{13} \mathrm{C} \mathrm{NMR}\left(\mathrm{CDCl}_{3}, 150 \mathrm{MHz}\right) \delta 199.9$, $111.9,109.1,83.3,82.3,77.8,75.9,27.4,27.0,26.8,26.3,18.3$; HRMS (CI) calcd for $\left[\mathrm{C}_{12} \mathrm{H}_{20} \mathrm{O}_{5}+\mathrm{Na}\right]^{+}: 267.1203$, Found: 267.1197.

(E)-ethyl 3-((2'R,3'S)-2',2'-dimethyl-5'-((4"R,5”S)-2",2",5”-trimethyl-1",3"dioxolan-4"-yl)-1,3-dioxolane-4'-yl)acrylate (13). 


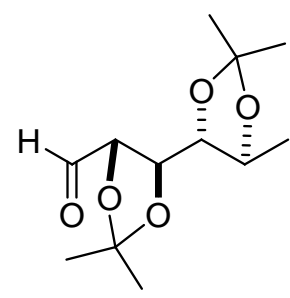

12

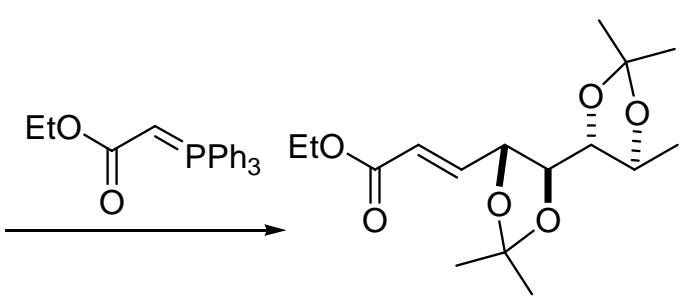

13

To a solution of aldehyde 12 (161 mg, $0.66 \mathrm{mmol})$ in $4 \mathrm{~mL}$ of $\mathrm{CH}_{2} \mathrm{Cl}_{2}$ was added yield (460 mg, $1.32 \mathrm{mmol}$ ) at $\mathrm{rt}$. In $2 \mathrm{~h}$, the solvent was removed in vacuo. The crude product was purified by flash chromatography on silica gel $(9: 1(\mathrm{v} / \mathrm{v})$ hexane/EtOAc) provided acetonide 13 (168 mg, 81\% yield) as a colorless oil. $\mathrm{R}_{f}=0.57$ (7:3 (v/v) hexane/EtOAc); IR (neat, $\left.\mathrm{cm}^{-1}\right)$ 2989, 1714; $[\alpha]^{25}{ }_{\mathrm{D}}+14.0\left(\mathrm{c} 1, \mathrm{CHCl}_{3}\right) ;{ }^{1} \mathrm{H}$ NMR $\left(\mathrm{CDCl}_{3}, 270 \mathrm{MHz}\right) \delta$ $6.99(\mathrm{dd}, J=15.6,4.3 \mathrm{~Hz}, 1 \mathrm{H}), 6.14(\mathrm{dd}, J=15.8,1.8 \mathrm{~Hz}, 1 \mathrm{H}), 4.56$ (ddd, $J=7.5,4.3$, $1.8 \mathrm{~Hz}, 1 \mathrm{H}), 4.19$ (q, $J=7.1 \mathrm{~Hz}, 2 \mathrm{H}), 4.01(\mathrm{dd}, J=7.5,6.2 \mathrm{~Hz}, 1 \mathrm{H}), 3.68$ (dd, $J=7.9,7.5$ $\mathrm{Hz}, 1 \mathrm{H}), 3.54$ (dd, $J=7.7,7.7 \mathrm{~Hz}, 1 \mathrm{H}), 1.40$ (s, 3H), 1.39 (s, 6H), 1.34 (d, J= 5.9 Hz, $3 \mathrm{H}), 1.33(\mathrm{~s}, 3 \mathrm{H}), 1.27$ (t, $J=7.1 \mathrm{~Hz}, 3 \mathrm{H}) ;{ }^{13} \mathrm{C} \mathrm{NMR}\left(\mathrm{CDCl}_{3}, 67.5 \mathrm{MHz}\right) \delta 166.3,145.0$, 121.4, 110.4, 109.1, 83.1, 81.5, 79.3, 76.6, 60.5, 27.4, 27.0, 26.9, 26.8, 18.5, 14.3; HRMS (CI) calcd for $\left[\mathrm{C}_{16} \mathrm{H}_{26} \mathrm{O}_{4}+\mathrm{Na}\right]^{+}: 337.1622$, Found: 337.1618.

\section{Ethyl (6S,7S)-4,5-dihydroxy-2,4-octdienoate (14a).}

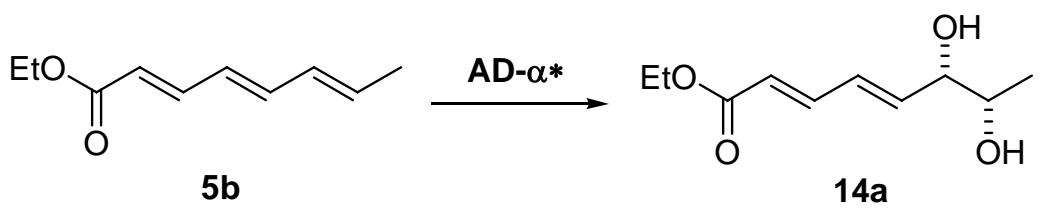

To a $500 \mathrm{~mL}$ round bottom flask was added 1:1 t-butyl alcohol $(160 \mathrm{~mL}) /$ water $(160 \mathrm{~mL})$, $\mathrm{K}_{3} \mathrm{Fe}(\mathrm{CN})_{6}(45.0 \mathrm{~g}, 136.8 \mathrm{mmol}), \mathrm{K}_{2} \mathrm{CO}_{3}(18.9 \mathrm{~g}, 136.8 \mathrm{mmol}), \mathrm{CH}_{3} \mathrm{SO}_{2} \mathrm{NH}_{2}(4.33 \mathrm{mg}$, $45.6 \mathrm{mmol}$ ), (DHQ) 2 -PHAL (702 mg, $2 \mathrm{~mol} \%$ ), $\mathrm{OsO}_{4}(116 \mathrm{mg}, 1 \mathrm{~mol} \%)$. The mixture was stirred at room temperature for $15 \mathrm{~min}$ and then cooled to $0{ }^{\circ} \mathrm{C}$. To this solution was added trienoate $5 \mathbf{b}(7.57 \mathrm{~g}, 45.6 \mathrm{mmol})$ in $10 \mathrm{~mL}$ of $t$-butyl alcohol/water: 1/1 and the reaction was stirred vigorously at $0{ }^{\circ} \mathrm{C}$ overnight. Sat. aqueous sodium sulfite $(70 \mathrm{~mL})$ was added to quench the reaction while stirring vigorously. Ethyl acetate $(80 \mathrm{~mL})$ was added to the reaction mixture, and after separation of the layers, the aqueous layer was further extracted with ethyl acetate $(3 \times 25 \mathrm{~mL})$. The combined organic layers were 
washed with $2 \mathrm{~N} \mathrm{KOH}(60 \mathrm{~mL})$ and brine to remove the methanesulfonamide, dried over anhydrous $\mathrm{Na}_{2} \mathrm{SO}_{4}$, and concentrated to afford the crude product. Flash chromatography on silica gel $(1: 1(\mathrm{v} / \mathrm{v})$ hexane/EtOAc) provided compound $14 \mathrm{a}(7.57 \mathrm{~g}, 83 \%$ yield) as a colorless oil. $\mathrm{R}_{f}=0.16\left(1: 1(\mathrm{v} / \mathrm{v})\right.$ hexane/EtOAc); IR (neat, $\left.\mathrm{cm}^{-1}\right) 3414,2982,1702$; $[\alpha]^{25}=-48^{\circ}\left(c 1, \mathrm{CHCl}_{3}\right) ;{ }^{1} \mathrm{H} \mathrm{NMR}\left(\mathrm{CDCl}_{3}, 270 \mathrm{MHz}\right) \delta 7.23(\mathrm{dd}, J=15.4,10.5 \mathrm{~Hz}$, 1H), $6.42(\mathrm{dd}, J=15.2,11.1 \mathrm{~Hz}, 1 \mathrm{H}), 6.05(\mathrm{dd}, J=15.2,6.2 \mathrm{~Hz}, 1 \mathrm{H}), 5.87$ (d, $J=15.4$ Hz, 1H), 4.17 (q, $J=7.1 \mathrm{~Hz}, 2 \mathrm{H}), 3.94$ (dd, $J=10.5,6.3 \mathrm{~Hz}, 1 \mathrm{H}), 3.64$ (dq, $J=10.2,6.3$ $\mathrm{Hz}, 1 \mathrm{H}), 3.00$ (bs, 1H), 2.76 (bs, 1H), 1.26 (t, $J=7.1 \mathrm{~Hz}, 3 \mathrm{H}), 1.16$ (d, $J=6.3 \mathrm{~Hz}, 3 \mathrm{H}$ );

${ }^{13} \mathrm{C}$ NMR $\left(\mathrm{CDCl}_{3}, 67.5 \mathrm{MHz}\right) \delta 167.0,143.6,140.7,129.6,122.2,70.6,60.5,18.9,14.3$, 14.0; HRMS (CI) calcd for $\left[\mathrm{C}_{10} \mathrm{H}_{16} \mathrm{O}_{4}+\mathrm{Na}\right]^{+}:$223.0941, Found: 223.0937.

\section{(2E,4E)-ethyl 5-((4'S,5'S)-2',2',5'-trimethyl-1',3'-dioxolan-4'-yl)penta-2,4-dienoate} (14).

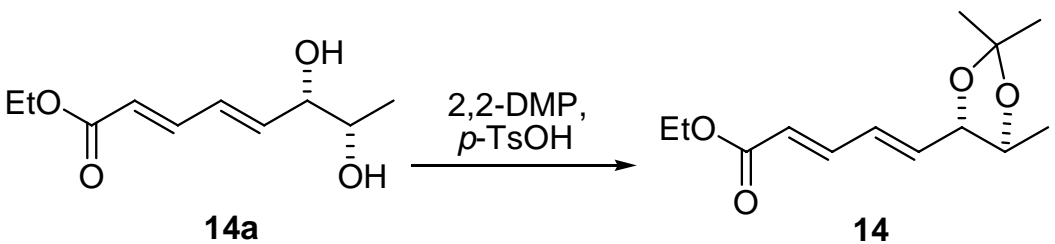

To a solution of diol 14a $(3.0 \mathrm{~g}, 15.0 \mathrm{mmol})$ in $150 \mathrm{~mL}$ acetone was added 2,2dimethoxypropane $(18.5 \mathrm{~mL}, 150 \mathrm{mmol})$ and $p$-toluenesulfonic acid monohydrate $(0.29 \mathrm{~g}$, $1.5 \mathrm{mmol}$ ) at $0{ }^{\circ} \mathrm{C}$. In an hour, the reaction was quenched by adding sat'd $\mathrm{NaHCO}_{3}$ and the mixture was filtered through a pad of celite. The aqueous layer was separated extracted with $\mathrm{Et}_{2} \mathrm{O}$, the combined organic layers were wished with brine, dried over anhydrous $\mathrm{Na}_{2} \mathrm{SO}_{4}$, and concentrated to afford the crude product. Flash chromatography on silica gel (9:1 (v/v) hexane/EtOAc) provided compound 14 (3.12 g, 87\% yield) as a colorless oil. $\mathrm{R}_{f}=0.63\left(7: 3(\mathrm{v} / \mathrm{v})\right.$ hexane/EtOAc); IR (neat, $\left.\mathrm{cm}^{-1}\right) 2984,1716 ;[\alpha]^{25}{ }_{\mathrm{D}}=$ $-2.1^{\circ}\left(\right.$ c $\left.1, \mathrm{CHCl}_{3}\right) ;{ }^{1} \mathrm{H}$ NMR $\left(\mathrm{CDCl}_{3}, 270 \mathrm{MHz}\right) \delta 7.22(\mathrm{dd}, J=15.6,10.9 \mathrm{~Hz}, 1 \mathrm{H}), 6.40$ (dd, $J=15.1,10.9 \mathrm{~Hz}, 1 \mathrm{H}), 5.98$ (dd, $J=15.1,6.8 \mathrm{~Hz}, 1 \mathrm{H}), 5.87(\mathrm{~d}, J=15.6 \mathrm{~Hz}, 1 \mathrm{H})$, $4.17(\mathrm{q}, J=7.2 \mathrm{~Hz}, 2 \mathrm{H}), 3.97(\mathrm{dd}, J=7.9,7.4 \mathrm{~Hz}, 1 \mathrm{H}), 3.78(\mathrm{dq}, J=8.4,5.9 \mathrm{~Hz}, 1 \mathrm{H})$, 1.40 (s, 3H), 1.39 (s, 3H), 1.26 (t, $J=7.2 \mathrm{~Hz}, 3 \mathrm{H}), 1.23$ (d, $J=6.4 \mathrm{~Hz}, 3 \mathrm{H}) ;{ }^{13} \mathrm{C}$ NMR $\left(\mathrm{CDCl}_{3}, 67.5 \mathrm{MHz}\right) \delta 166.5,142.9,137.6,130.4,122.4,108.7,82.6,76.5,60.2,27.1$, 
26.6, 16.4, 14.1; HRMS (CI) calcd for $\left[\mathrm{C}_{13} \mathrm{H}_{20} \mathrm{O}_{4}+\mathrm{Na}\right]^{+}: 263.1254$, Found: 263.1241.

(E,2S,3R)-ethyl 2,3-dihydroxy-5-((4'S,5'S)-2',2',5'-trimethyl-1',3'-dioxolan-4'yl)pent-4-enoate (15a) and (E,2R,3S)-ethyl 4,5-dihydroxy-5-((4'S,5'S)-2',2',5'trimethyl-1',3'-dioxolan-4'-yl)pent-2-enoate (15b).

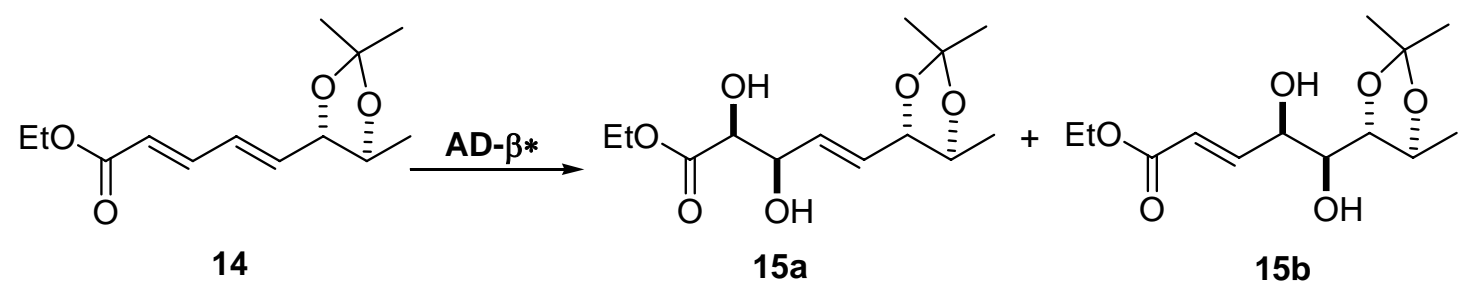

To a $250 \mathrm{~mL}$ round bottom flask was added 1:1 t-butyl alcohol $(50 \mathrm{~mL}) / \mathrm{H}_{2} \mathrm{O}(50 \mathrm{~mL})$, $\mathrm{K}_{3} \mathrm{Fe}(\mathrm{CN})_{6}(7.9 \mathrm{~g}, 24.2 \mathrm{mmol}), \mathrm{K}_{2} \mathrm{CO}_{3}(3.34 \mathrm{mg}, 24.2 \mathrm{mmol}), \mathrm{CH}_{3} \mathrm{SO}_{2} \mathrm{NH}_{2}(765 \mathrm{mg}, 8.05$ mmol), (DHQD) $)_{2}$-PHAL (260 mg, $0.32 \mathrm{mmol}, 4 \mathrm{~mol} \%$ ) and $\mathrm{OsO}_{4}(41 \mathrm{mg}, 0.16 \mathrm{mmol}, 2$ mol\%). The mixture was stirred at room temperature for $15 \mathrm{~min}$ and then cooled to $0{ }^{\circ} \mathrm{C}$. To this solution was added acetonide $14(1.93 \mathrm{~g}, 8.05 \mathrm{mmol})$ dropwise and the reaction was stirred vigorously at $0{ }^{\circ} \mathrm{C}$ overnight. Sat. aqueous sodium sulfite $(50 \mathrm{~mL})$ was added to quench the reaction while stirring vigorously. Ethyl acetate $(20 \mathrm{~mL})$ was added to the reaction mixture, and after separation of the layers, the aqueous layer was further extracted with ethyl acetate $(3 \times 25 \mathrm{~mL})$. The combined organic layers were washed with $2 \mathrm{~N} \mathrm{KOH}(40 \mathrm{~mL})$ and brine $(20 \mathrm{~mL})$ to remove the methanesulfonamide, dried over anhydrous $\mathrm{Na}_{2} \mathrm{SO}_{4}$, and concentrated to afford the crude mixture of regioisomers $\left(\mathbf{1 5 a} / \mathbf{1 5 b}=1 / 1\right.$ determined by ${ }^{1} \mathrm{H}$ NMR). Flash chromatography on silica gel $(1: 1(\mathrm{v} / \mathrm{v})$ hexane/EtOAc) provided the two pure regioisomers $1.86 \mathrm{~g}, 85 \%$ combined yield. 15a (929 mg, 42.5\% yield), a colorless oil. 15b (929 mg, 42.5\%) a white solid.

15a: $\mathrm{R}_{f}=0.10\left(1: 1(\mathrm{v} / \mathrm{v})\right.$ hexane/EtOAc); IR $\left(\right.$ neat, $\left.\mathrm{cm}^{-1}\right) 3446,2983,1762 ;[\alpha]^{25} \mathrm{D}+27.9$ (c 1, $\left.\mathrm{CHCl}_{3}\right) ;{ }^{1} \mathrm{H} \mathrm{NMR}\left(\mathrm{CDCl}_{3}, 270 \mathrm{MHz}\right) \delta 5.88(\mathrm{dd}, J=15.4,5.7 \mathrm{~Hz}, 1 \mathrm{H}), 5.70(\mathrm{ddd}, J$ $=15.6,7.1,1.2 \mathrm{~Hz}, 1 \mathrm{H}), 4.38(\mathrm{bs}, 1 \mathrm{H}), 4.19$ (q, $J=7.1 \mathrm{~Hz}, 2 \mathrm{H}), 4.08$ (dd, $J=6.1,3.0 \mathrm{~Hz}$, $1 \mathrm{H}), 3.88$ (dd, $J=7.9,7.5 \mathrm{~Hz}, 1 \mathrm{H}), 3.70(\mathrm{dd}, J=8.5,5.9 \mathrm{~Hz}, 1 \mathrm{H}), 3.62(\mathrm{~d}, J=6.3 \mathrm{~Hz}$, $1 \mathrm{H}), 3.23$ (dd, $J=4.9,4.0 \mathrm{~Hz}, 1 \mathrm{H}), 2.71$ (dd, $J=8.1,7.9 \mathrm{~Hz}, 1 \mathrm{H}), 1.34$ (s, 3H), 1.32 (s, $3 \mathrm{H}), 1.23(\mathrm{t}, J=7.1 \mathrm{~Hz}, 3 \mathrm{H}), 1.18(\mathrm{~d}, J=5.9 \mathrm{~Hz}, 3 \mathrm{H}) ;{ }^{13} \mathrm{C} \mathrm{NMR}\left(\mathrm{CDCl}_{3}, 67.5 \mathrm{MHz}\right) \delta$ 172.4, 132.9, 128.8, 108.3, 82.8, 76.5, 73.5, 72.4, 61.8, 27.1, 26.7, 16.2, 13.9; HRMS (CI) 
calcd for $\left[\mathrm{C}_{13} \mathrm{H}_{22} \mathrm{O}_{4}+\mathrm{Na}\right]^{+}:$297.1309, Found: 297.1294. 15b $\mathrm{R}_{f}=0.14(1: 1(\mathrm{v} / \mathrm{v})$ hexane/EtOAc); IR (neat, $\left.\mathrm{cm}^{-1}\right) 3298,2983,1723 ;[\alpha]^{25}+9.8\left(c 1, \mathrm{CHCl}_{3}\right) ;{ }^{1} \mathrm{H}$ NMR $\left(\mathrm{CDCl}_{3}, 270 \mathrm{MHz}\right) \delta 7.01(\mathrm{dd}, J=15.6,4.2 \mathrm{~Hz}, 1 \mathrm{H}), 6.18(\mathrm{dd}, J=15.6,2.0 \mathrm{~Hz}, 1 \mathrm{H})$, 4.56 (ddd, $J=7.9,4.2,2.2 \mathrm{~Hz}, 1 \mathrm{H}$ ), 4.21 (q, $J=7.2 \mathrm{~Hz}, 2 \mathrm{H}$ ), 4.09 (ddd, $J=13.1,6.2,6.0$ $\mathrm{Hz} 1 \mathrm{H}), 3.70-3.62(\mathrm{~m}, 2 \mathrm{H}), 2.77$ (d, $J=5.9 \mathrm{~Hz}, 1 \mathrm{H}), 2.39$ (d, $J=6.7 \mathrm{~Hz}, 1 \mathrm{H}), 1.42(\mathrm{~s}$, $3 \mathrm{H}), 1.39$ (s, $3 \mathrm{H}), 1.38(\mathrm{~d}, J=5.9 \mathrm{~Hz}, 3 \mathrm{H}), 1.30(\mathrm{t}, J=7.2 \mathrm{~Hz}, 3 \mathrm{H}) ;{ }^{13} \mathrm{C} \mathrm{NMR}\left(\mathrm{CDCl}_{3}\right.$, $67.5 \mathrm{MHz}) \delta 166.4,147.0,122.2,108.7,81.7,75.9,74.0,70.7,60.6,27.3,26.9,19.1$, 14.2; HRMS (CI) calcd for $\left[\mathrm{C}_{13} \mathrm{H}_{22} \mathrm{O}_{4}+\mathrm{Na}\right]^{+}: 297.1309$, Found: 297.1313.

(E)-ethyl 3-((4'R,5'S)-2',2'-dimethyl-5'-((4”R,5”S)-2",2",5”-trimethyl-1",3”dioxolan-4”-yl)-1',3'-dioxolane-4'-yl)acrylate (13).

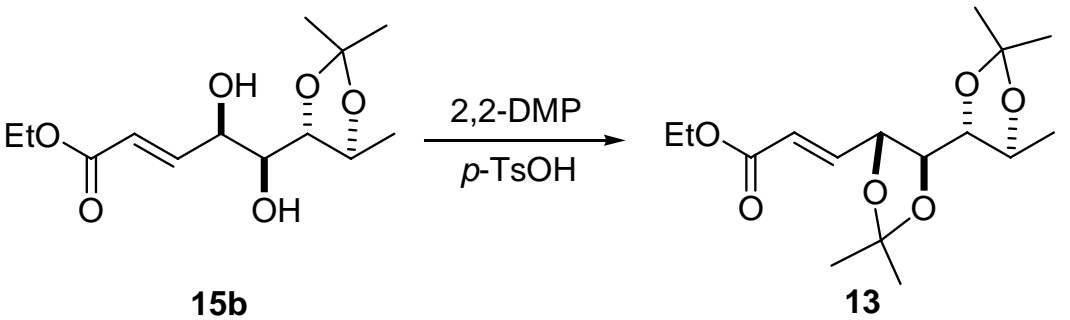

To a solution of diol 15b (405 mg, $1.48 \mathrm{~mL})$ in $25 \mathrm{~mL}$ acetone was added 2,2dimethoxypropane $(1.9 \mathrm{~mL}, 14.8 \mathrm{mmol})$ and $p$-toluenesulfonic acid monohydrate $(29 \mathrm{mg}$, $0.15 \mathrm{mmol}$ ) at $0{ }^{\circ} \mathrm{C}$. In two hour, the reaction was quenched by adding sat'd $\mathrm{NaHCO}_{3}$. Then $15 \mathrm{~mL} \mathrm{Et}_{2} \mathrm{O}$ and $10 \mathrm{~mL} \mathrm{H}_{2} \mathrm{O}$ was added. The aqueous layer was extracted with $\mathrm{Et}_{2} \mathrm{O}$. The combined organic layers were wished with brine and dried over anhydrous $\mathrm{Na}_{2} \mathrm{SO}_{4}$, and concentrated to afford the crude product. Flash chromatography on silica gel (9:1 (v/v) hexane/EtOAc) provided acetonide $13(440 \mathrm{mg}, 95 \%)$ as a colorless oil. $\mathrm{R}_{f}=$ 0.57 (7:3 (v/v) hexane/EtOAc); IR (neat, $\left.\mathrm{cm}^{-1}\right)$ 2989, 1714; $[\alpha]^{25}+14.0\left(\mathrm{c} 1, \mathrm{CHCl}_{3}\right) ;{ }^{1} \mathrm{H}$ NMR $\left(\mathrm{CDCl}_{3}, 270 \mathrm{MHz}\right) \delta 6.99(\mathrm{dd}, J=15.6,4.3 \mathrm{~Hz}, 1 \mathrm{H}), 6.14(\mathrm{dd}, J=15.8,1.8 \mathrm{~Hz}$, 1H), 4.56 (ddd, $J=7.5,4.3,1.8 \mathrm{~Hz}, 1 \mathrm{H}), 4.19$ (q, $J=7.1 \mathrm{~Hz}, 2 \mathrm{H}), 4.01$ (dd, $J=7.5,6.2$ $\mathrm{Hz}, 1 \mathrm{H}), 3.68(\mathrm{dd}, J=7.9,7.5 \mathrm{~Hz}, 1 \mathrm{H}), 3.54(\mathrm{dd}, J=7.7,7.7 \mathrm{~Hz}, 1 \mathrm{H}), 1.40(\mathrm{~s}, 3 \mathrm{H}), 1.39$ $(\mathrm{s}, 6 \mathrm{H}), 1.34(\mathrm{~d}, J=5.9 \mathrm{~Hz}, 3 \mathrm{H}), 1.33(\mathrm{~s}, 3 \mathrm{H}), 1.27(\mathrm{t}, J=7.1 \mathrm{~Hz}, 3 \mathrm{H}) ;{ }^{13} \mathrm{C} \mathrm{NMR}\left(\mathrm{CDCl}_{3}\right.$, 67.5 MHz) $\delta 166.3,145.0,121.4,110.4,109.1,83.1,81.5,79.3,76.6,60.5,27.4,27.0$, 26.9, 26.8, 18.5, 14.3; HRMS (CI) calcd for $\left[\mathrm{C}_{16} \mathrm{H}_{26} \mathrm{O}_{4}+\mathrm{Na}\right]^{+}: 337.1622$, Found: 
337.1618 .

(E)-3-((4'R,5'S)-2',2'-dimethyl-5'-((4"R,5”S)-2",2",5"-trimethyl-1",3"-dioxolan-4"yl)-1',3'-dioxolane-4'-yl)prop-2-en-1-ol (13a).
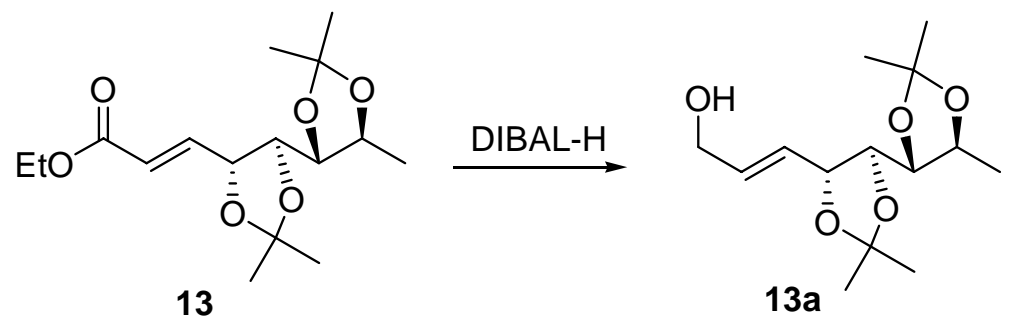

To a solution of ester 13 (413 mg, $1.32 \mathrm{mmol})$ in $7 \mathrm{~mL}$ of THF was added DIBAL-H (4.9 $\mathrm{ml}, 1.0 \mathrm{M}$ in hexanes, $4.0 \mathrm{mmol}$ ) dropwise at $-78{ }^{\circ} \mathrm{C}$. In $30 \mathrm{~min}$, the reaction was quenched by adding $3 \mathrm{~mL}$ of acetone and $10 \mathrm{~mL}$ of $10 \% \mathrm{NaOH}$ solution. The mixture was filtered through a pad of celite. The aqueous layer was extracted with $\mathrm{Et}_{2} \mathrm{O}$. The combined organic layers were wished with brine and dried over anhydrous $\mathrm{Na}_{2} \mathrm{SO}_{4}$, and concentrated to afford the crude product. Flash chromatography on silica gel $(7: 3(\mathrm{v} / \mathrm{v})$ hexane/EtOAc) provided allylic alcohol 13a $\left(317 \mathrm{mg}, 89 \%\right.$ yield) as a colorless oil. $\mathrm{R}_{f}=$ 0.14 (7:3 (v/v) hexane/EtOAc); IR (neat, $\left.\mathrm{cm}^{-1}\right)$ 3427, 2982; $[\alpha]^{25}{ }_{\mathrm{D}}+8.8\left(\mathrm{c} 1, \mathrm{CHCl}_{3}\right) ;{ }^{1} \mathrm{H}$ NMR $\left(\mathrm{CDCl}_{3}, 270 \mathrm{MHz}\right) \delta 5.99$ (dd, $\left.J=15.5,4.9,0.8 \mathrm{~Hz}, 1 \mathrm{H}\right), 5.76$ (ddd, $J=15.4,6.1$, 1.6, $1.4 \mathrm{~Hz}, 1 \mathrm{H}), 4.44$ (dd, $J=7.1,6.5 \mathrm{~Hz}, 1 \mathrm{H}), 4.15-4.14$ (m, 2H), 4.02 (dq, $J=7.7,5.9$ Hz, 1H), 3.69 (dd, $J=7.1,7.1 \mathrm{~Hz}, 1 \mathrm{H}$ ), 3.57 (dd, $J=7.7,6.9 \mathrm{~Hz}, 1 \mathrm{H}$ ), 2.05 (bs, 1H), 1.39 (s, 3H), 1.38 (s, 6H), 1.33 (s, 3H), $1.32(\mathrm{~d}, J=5.9 \mathrm{~Hz}, 3 \mathrm{H}) ;{ }^{13} \mathrm{C} \mathrm{NMR}\left(\mathrm{CDCl}_{3}, 67.5 \mathrm{MHz}\right)$ $\delta$ 132.6, 128.3, 109.4, 108.8, 82.6, 81.3, 79.5, 75.6, 62.6, 27.3, 26.9, 26.9, 26.8, 18.6; HRMS (CI) calcd for $\left[\mathrm{C}_{14} \mathrm{H}_{24} \mathrm{O}_{5}+\mathrm{Na}\right]^{+}: 295.1516$, Found: 295.1514.

(E)-3-((4'R,5'S)-2',2'-dimethyl-5'-((4"R,5”S)-2",2",5"-trimethyl-1",3"-dioxolan-4"yl)-1',3'-dioxolane-4'-yl)acrylaldehyde (9). 


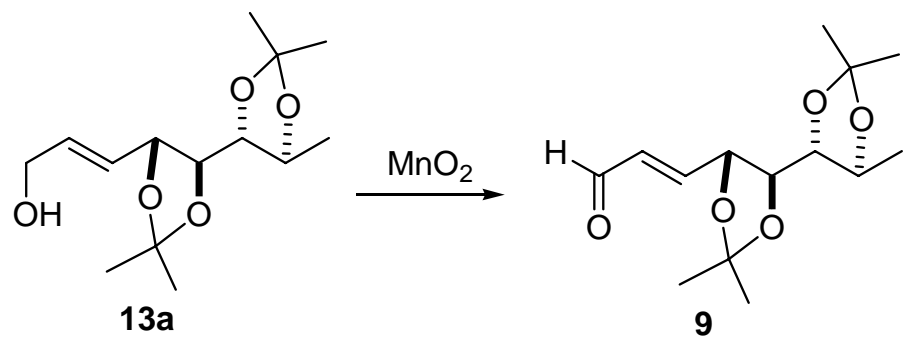

To a solution of alcohol 13a ( $240 \mathrm{mg}, 0.88 \mathrm{mmol}$ ) in $9 \mathrm{~mL}$ of $\mathrm{CH}_{2} \mathrm{Cl}_{2}$ was added $\mathrm{MnO}_{2}$ (767 mg, $8.8 \mathrm{mmol}$ ) at room temperture. In $12 \mathrm{~h}$, the reaction mixture was filtered through a pad of celite and washed with EtOAc. The organic layers were wished with brine and dried over anhydrous $\mathrm{Na}_{2} \mathrm{SO}_{4}$, and concentrated to afford the crude product. Flash chromatography on silica gel $(8: 2(\mathrm{v} / \mathrm{v})$ hexane/EtOAc) provided aldehyde 9 (234 mg, $92 \%$ yield) as a white solid. $\mathrm{R}_{f}=0.53\left(7: 3(\mathrm{v} / \mathrm{v})\right.$ hexane/EtOAc); IR (neat, $\mathrm{cm}^{-1}$ ) 2989, 1682; $[\alpha]_{\mathrm{D}}^{25}+36.1\left(c \mathrm{1}, \mathrm{CHCl}_{3}\right) ;{ }^{1} \mathrm{H} \mathrm{NMR}\left(\mathrm{CDCl}_{3}, 270 \mathrm{MHz}\right) \delta 9.59(\mathrm{~d}, J=7.9$, $\mathrm{Hz}, 1 \mathrm{H}$ ), $\delta 6.88(\mathrm{dd}, J=15.8,4.0 \mathrm{~Hz}, 1 \mathrm{H}), 6.45$ (ddd, $J=15.8,7.9,1.6 \mathrm{~Hz}, 1 \mathrm{H}), 4.66$ (ddd, $J=7.7,3.8,1.6, \mathrm{~Hz}, 1 \mathrm{H}), 4.02(\mathrm{dq}, J=7.5,5.9 \mathrm{~Hz}, 1 \mathrm{H}), 3.68(\mathrm{dd}, J=8.1,7.9 \mathrm{~Hz}$, $1 \mathrm{H}), 3.55$ (dd, $J=8.1,7.7 \mathrm{~Hz}, 1 \mathrm{H}), 1.42$ (s, 3H), 1.41 (s, 3H), 1.40 (s, 3H), 1.36 (d, $J=$ $6.1 \mathrm{~Hz}, 3 \mathrm{H}), 1.33(\mathrm{~s}, 3 \mathrm{H}) ;{ }^{13} \mathrm{C} \mathrm{NMR}\left(\mathrm{CDCl}_{3}, 67.5 \mathrm{MHz}\right) \delta 193.3,153.5,131.3,110.7$, 109.1, 83.0, 81.5, 79.5, 76.8, 27.3, 26.8, 26.8, 26.5, 18.3; HRMS (CI) calcd for $\left[\mathrm{C}_{14} \mathrm{H}_{22} \mathrm{O}_{5}\right.$ $+\mathrm{Na}]^{+}: 293.1359$, Found: 293.1366.

\section{(3R,E)-1-((4'R,5'S)-2',2'-dimethyl-5'-((4"R,5”S)-2",2",5"-trimethyl-1",3"-dioxolan-} 4"-yl)-1',3'-dioxolan-4'-yl)hexa-1,5-dien-3-ol (17a).

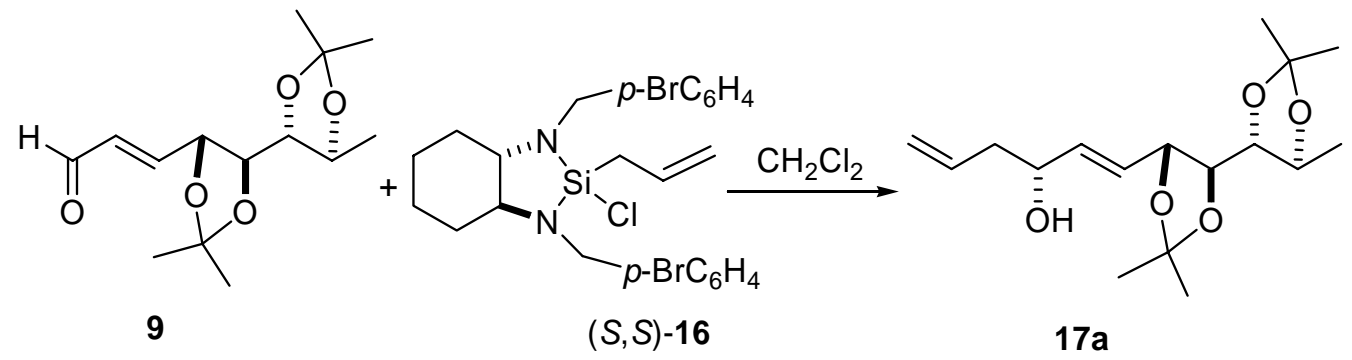

To a solution of (S,S)-16 (690 mg, $1.25 \mathrm{mmol})$ in $4.25 \mathrm{~mL}$ of $\mathrm{CH}_{2} \mathrm{Cl}_{2}$ was added aldehyde 9 (135 mg, $0.5 \mathrm{mmol})$ in $2 \mathrm{~mL}$ of $\mathrm{CH}_{2} \mathrm{Cl}_{2}$ dropwise at $-10{ }^{\circ} \mathrm{C}$. The reaction flask was put in a freezer $\left(-10{ }^{\circ} \mathrm{C}\right)$. In $36 \mathrm{~h}$, the reaction was quenched by adding $1 \mathrm{~N} \mathrm{HCl}$ and EtOAc, and the mixture was vigorously stirred at room temperature for $15 \mathrm{~min}$. The mixture was 
filtered through a pad of celite and the layers were separated. The aqueous layer was extracted with EtOAc. The combined organic layers were wished with brine, dried over anhydrous $\mathrm{Na}_{2} \mathrm{SO}_{4}$, and concentrated to afford the crude product. Flash chromatography on silica gel (9:1 (v/v) hexane/EtOAc) provided compound 17a (143 mg, 92\% yield) as a white solid. $\mathrm{R}_{f}=0.23\left(7: 3(\mathrm{v} / \mathrm{v})\right.$ hexane/EtOAc); IR (neat, $\left.\mathrm{cm}^{-1}\right) 3453,2989,1637 ;[\alpha]^{25}{ }_{\mathrm{D}}$ $+13.8\left(\right.$ c $\left.1, \mathrm{CHCl}_{3}\right) ;{ }^{1} \mathrm{H} \mathrm{NMR}\left(\mathrm{CDCl}_{3}, 600 \mathrm{MHz}\right) \delta 5.90(\mathrm{ddd}, J=15.6,5.4,1.2 \mathrm{~Hz}, 1 \mathrm{H})$, $\delta 5.80$ (dddd, $J=16.8,10.8,7.2,7.2 \mathrm{~Hz}, 1 \mathrm{H}$ ), 5.77 (ddd, 15.6, 6.6, $1.2 \mathrm{~Hz}, 1 \mathrm{H}$ ), 5.15-5.11 (m, 2H), 4.44 (dd, $J=7.2,6.6 \mathrm{~Hz}, 1 \mathrm{H}), 4.23(\mathrm{dq}, J=5.4,1.2 \mathrm{~Hz}, 1 \mathrm{H}), 4.03$ (ddd, $J=10.2$, 7.8, $5.4 \mathrm{~Hz}, 1 \mathrm{H}), 3.68(\mathrm{dd}, J=7.2,7.2 \mathrm{~Hz}, 1 \mathrm{H}), 3.58(\mathrm{dd}, J=7.8,7.2 \mathrm{~Hz}, 1 \mathrm{H}), 2.34$ (ddddd, $J=12.6,7.2,5.4,1.2,1.2 \mathrm{~Hz}, 1 \mathrm{H}$ ), 2.28 (ddddd, $J=13.8,7.2,7.2,1.2,1.2 \mathrm{~Hz}$, 1H), 1.40 (s, 3H), 1.39 (s, 3H), 1.39 (s, 3H), 1.33 (s, 3H), 1.33 (d, $J=6.6 \mathrm{~Hz}, 3 \mathrm{H}) ;{ }^{13} \mathrm{C}$ NMR $\left(\mathrm{CDCl}_{3}, 67.5 \mathrm{MHz}\right) \delta 135.4,133.9,127.9,118.3,109.4,108.7,82.6,81.4,79.7$, 75.7, 70.5, 41.5, 27.3, 26.9, 26.9, 26.8, 18.5; HRMS (CI) calcd for $\left[\mathrm{C}_{17} \mathrm{H}_{28} \mathrm{O}_{5}+\mathrm{Na}\right]^{+}$: 335.1829, Found: 335.1846.

\section{(3S,E)-1-((4'R,5'S)-2,2-dimethyl-5'-((4"R,5”S)-2",2",5”-trimethyl-1",3"-dioxolan- 4'-yl)-1',3'-dioxolan-4'-yl)hexa-1,5-dien-3-ol (17b).}

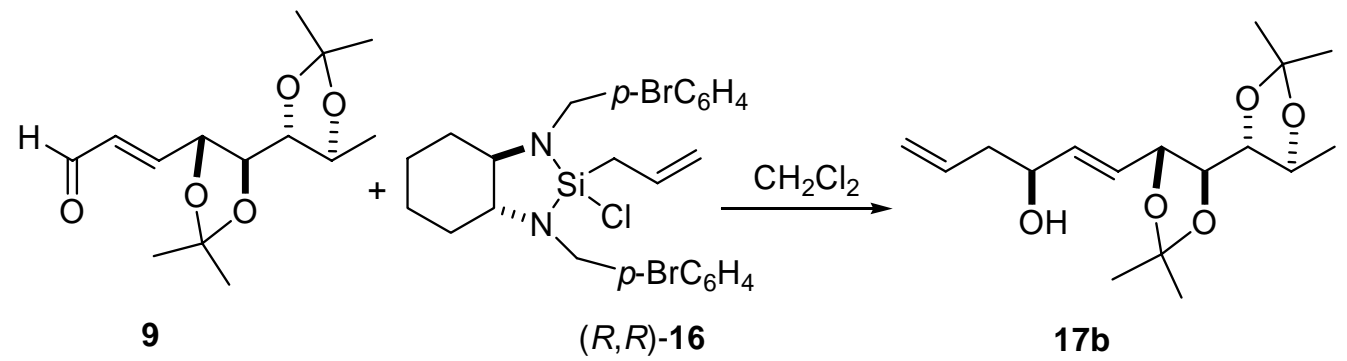

To a solution of $(\boldsymbol{R}, \boldsymbol{R})-\mathbf{1 6}(690 \mathrm{mg}, 1.25 \mathrm{mmol})$ in $4.25 \mathrm{~mL}$ of $\mathrm{CH}_{2} \mathrm{Cl}_{2}$ was added aldehyde 9 (135 mg, $0.5 \mathrm{mmol})$ in $2 \mathrm{~mL}$ of $\mathrm{CH}_{2} \mathrm{Cl}_{2}$ dropwise at $-10{ }^{\circ} \mathrm{C}$. The reaction flask was put in a freezer $\left(-10{ }^{\circ} \mathrm{C}\right)$. In $38 \mathrm{~h}$, the reaction was quenched by adding $1 \mathrm{~N}$ $\mathrm{HCl}$ and EtOAc, and the mixture was vigorously stirred at room temperature for $15 \mathrm{~min}$. The mixture was filtered through a pad of celite and the layers were separated. The aqueous layer was extracted with EtOAc. The combined organic layers were wished with brine, dried over anhydrous $\mathrm{Na}_{2} \mathrm{SO}_{4}$, and concentrated to afford the crude product. Flash chromatography on silica gel (9:1 (v/v) hexane/EtOAc) provided compound 17b (148 mg, 
95\% yield) as a colorless oil. IR (neat, $\left.\mathrm{cm}^{-1}\right) 3491,2989,1642 ;[\alpha]^{25}{ }_{\mathrm{D}}+6.5\left(\mathrm{c} \mathrm{2}, \mathrm{CHCl}_{3}\right)$; ${ }^{1} \mathrm{H} \mathrm{NMR}\left(\mathrm{CDCl}_{3}, 600 \mathrm{MHz}\right) \delta 5.86(\mathrm{dd}, 15.6,6.0,1 \mathrm{H}), 5.78$ (dddd, $J=17.4,10.2,7.2$, $7.2 \mathrm{~Hz}, 1 \mathrm{H}), 5.73(\mathrm{dd}, 15.0,6.0 \mathrm{~Hz} 1 \mathrm{H}), 5.13-5.09(\mathrm{~m}, 2 \mathrm{H}), 4.42(\mathrm{dd}, J=7.2,6.6 \mathrm{~Hz}, 1 \mathrm{H})$, $4.18(\mathrm{dd}, J=12.6,6.0 \mathrm{~Hz}, 1 \mathrm{H}), 4.01$ (dq, $J=7.8,6.0 \mathrm{~Hz}, 1 \mathrm{H}), 3.67$ (dd, $J=7.2,7.2 \mathrm{~Hz}$, $1 \mathrm{H}), 3.56(\mathrm{dd}, J=7.8,7.2 \mathrm{~Hz}, 1 \mathrm{H}), 2.34-2.26(\mathrm{~m}, 2 \mathrm{H}), 1.39(\mathrm{~s}, 3 \mathrm{H}), 1.38(\mathrm{~s}, 3 \mathrm{H}), 1.38(\mathrm{~s}$, $3 \mathrm{H}), 1.32(\mathrm{~s}, 3 \mathrm{H}), 1.32(\mathrm{~d}, J=6.0 \mathrm{~Hz}, 3 \mathrm{H}) ;{ }^{13} \mathrm{C} \mathrm{NMR}\left(\mathrm{CDCl}_{3}, 67.5 \mathrm{MHz}\right) \delta 135.5,133.9$, 128.4, 118.3, 109.5, 108.8, 82.6, 81.3, 79.7, 75.7, 71.0, 41.6, 27.3, 26.9, 26.9, 26.8, 18.5; HRMS (CI) calcd for $\left[\mathrm{C}_{17} \mathrm{H}_{28} \mathrm{O}_{5}+\mathrm{Na}\right]^{+}: 335.1829$, Found: 335.1812.

\section{(3R,E)-1-((4'R,5'S)-2',2'-dimethyl-5'-((4”R,5”S)-2”,2”,5”-trimethyl-1",3”-dioxolan-} 4’-yl)-1',3'-dioxolan-4'-yl)hexa-1,5-dien-3-yl acrylate (8).

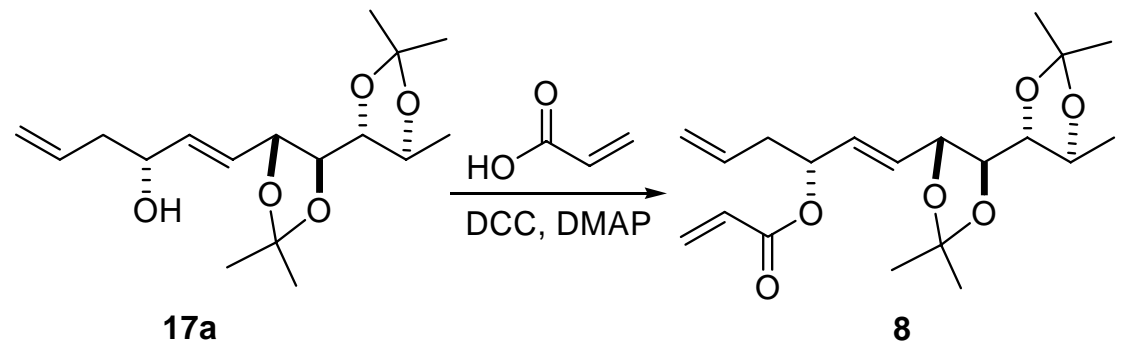

To a solution of alcohol 17a (116 mg, $0.37 \mathrm{mmol})$ in $2 \mathrm{~mL}$ of $\mathrm{CH}_{2} \mathrm{Cl}_{2}$ was added acrylic acid (0.11 mL, $1.49 \mathrm{mmol})$, DCC (306 mg, $1.49 \mathrm{mmol})$ and DMAP (5 mg, catalytic amount). In $4 \mathrm{~h}$, the reaction mixture was diluted with $\mathrm{Et}_{2} \mathrm{O}$ and filtered through a pad of celite and washed with $\mathrm{Et}_{2} \mathrm{O}$. The organic layer was washed with sat'd aqueous $\mathrm{NaHSO}_{4}$, sat'd aqueous $\mathrm{NaHCO}_{3}$, brine and dried over anhydrous $\mathrm{Na}_{2} \mathrm{SO}_{4}$, concentrated to afford the crude product. Flash chromatography on silica gel $(9: 1(\mathrm{v} / \mathrm{v})$ hexane/EtOAc) provided ester 8 (108 mg, 78\% yield) as a colorless oil. $R_{f}=0.69(7: 3(\mathrm{v} / \mathrm{v})$ hexane/EtOAc); IR $\left(\right.$ neat, $\left.\mathrm{cm}^{-1}\right) 2987,1736 ;[\alpha]^{25}+24.1\left(c 1, \mathrm{CHCl}_{3}\right) ;{ }^{1} \mathrm{H} \mathrm{NMR}\left(\mathrm{CDCl}_{3}, 270 \mathrm{MHz}\right) \delta 6.39$ (dd, $J=17.3,1.5 \mathrm{~Hz}, 1 \mathrm{H}$ ), 6.15 (dd, $J=17.3,10.1 \mathrm{~Hz}, 1 \mathrm{H}), 5.84-5.67$ (m, 4H), 5.45-5.39 (m, 1H), 5.13-5.04 (m, 2H), $4.44(\mathrm{dd}, J=7.4,2.5 \mathrm{~Hz}, 1 \mathrm{H}), 4.01$ (ddd, $J=13.3,6.2,5.9$ $\mathrm{Hz}, 1 \mathrm{H}), 3.64$ (dd, $J=7.4,7.2 \mathrm{~Hz}, 1 \mathrm{H}), 3.55$ (dd, $J=7.4,7.4 \mathrm{~Hz}, 1 \mathrm{H}), 2.46-2.41(\mathrm{~m}, 2 \mathrm{H})$, $1.40(\mathrm{~s}, 6 \mathrm{H}), 1.38(\mathrm{~s}, 3 \mathrm{H}), 1.33(\mathrm{~d}, J=6.0 \mathrm{~Hz}, 3 \mathrm{H}), 1.30(\mathrm{~s}, 3 \mathrm{H}) ;{ }^{13} \mathrm{C} \mathrm{NMR}\left(\mathrm{CDCl}_{3}, 67.5\right.$ MHz) $\delta 165.1,132.9,130.8,130.7,129.9,128.6,118.1,109.6,108.8,82.7,81.6,79.5$, 76.0, 72.9, 38.8, 27.3, 26.9 (2C), 26.7, 18.4; HRMS (CI) calcd for $\left[\mathrm{C}_{20} \mathrm{H}_{30} \mathrm{O}_{6}+\mathrm{Na}\right]^{+}$: 
389.1935, Found: 389.1928.

(3S,E)-1-((4'R,5'S)-2',2'-dimethyl-5'-((4"R,5"S)-2",2",5"-trimethyl-1",3"-dioxolan4"-yl)-1',3'-dioxolan-4'-yl)hexa-1,5-dien-3-yl acrylate (8b).

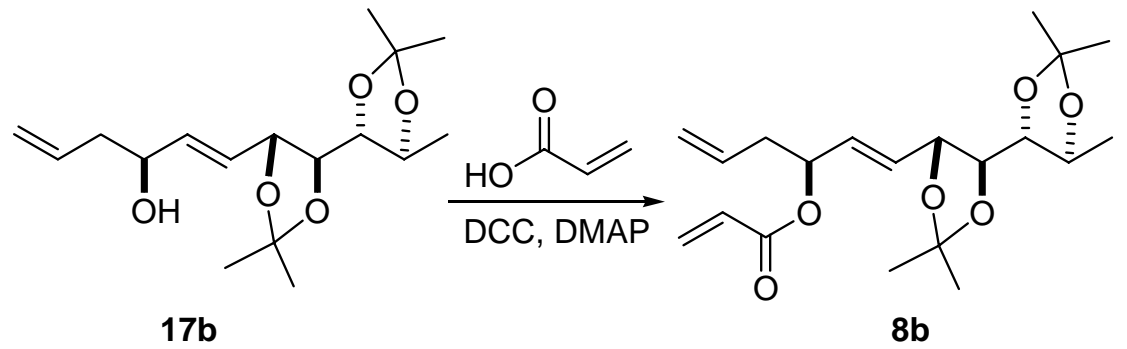

To a solution of alcohol $\mathbf{1 7 b}(120 \mathrm{mg}, 0.39 \mathrm{mmol})$ in $5 \mathrm{~mL}$ of $\mathrm{CH}_{2} \mathrm{Cl}_{2}$ was added acrylic acid $(0.12 \mathrm{~mL}, 1.55 \mathrm{mmol})$, DCC (320 $\mathrm{mg}, 1.55 \mathrm{mmol})$ and DMAP $(5 \mathrm{mg}$, catalytic amount). In $3 \mathrm{~h}$, the reaction mixture was diluted with $\mathrm{Et}_{2} \mathrm{O}$ and filtered through a pad of celite and washed with $\mathrm{Et}_{2} \mathrm{O}$. The organic layer was washed with sat'd aqueous $\mathrm{NaHSO}_{4}$, sat'd aqueous $\mathrm{NaHCO}_{3}$, brine and dried over anhydrous $\mathrm{Na}_{2} \mathrm{SO}_{4}$, concentrated to afford the crude product. Flash chromatography on silica gel $(9: 1(\mathrm{v} / \mathrm{v})$ hexane/EtOAc) provided ester $8 \mathbf{b}$ (106 mg, 76\% yield) as a colorless oil. $\mathrm{R}_{f}=0.68$ (7:3 (v/v) hexane/EtOAc); IR (neat, $\left.\mathrm{cm}^{-1}\right)$ 2988, 1739; $[\alpha]_{\mathrm{D}}^{25}-18.5\left(\mathrm{c} 1, \mathrm{CHCl}_{3}\right) ;{ }^{1} \mathrm{H} \mathrm{NMR}\left(\mathrm{CDCl}_{3}, 270 \mathrm{MHz}\right) \delta 6.37$ (dd, $J=17.3,1.5 \mathrm{~Hz}, 1 \mathrm{H}$ ), 6.08 (dd, $J=17.3,10.4 \mathrm{~Hz}, 1 \mathrm{H}), 5.85-5.62(\mathrm{~m}, 3 \mathrm{H}), 5.37$ (dt, $J=11.6,6.2 \mathrm{~Hz}, 1 \mathrm{H}), 5.10-5.03(\mathrm{~m}, 2 \mathrm{H}), 4.40(\mathrm{dd}, J=6.4,6.2 \mathrm{~Hz}, 2 \mathrm{H}), 4.06-3.96(\mathrm{~m}$, $1 \mathrm{H}), 3.63(\mathrm{dd}, J=7.4,7.2 \mathrm{~Hz}, 1 \mathrm{H}), 3.54(\mathrm{~d}, J=7.4,7.4 \mathrm{~Hz}, 1 \mathrm{H}), 2.69-2.64(\mathrm{~m}, 1 \mathrm{H})$, 2.40-2.33 (m, 1H), $1.38(\mathrm{~s}, 9 \mathrm{H}), 1.32(\mathrm{~d}, J=5.7 \mathrm{~Hz}, 3 \mathrm{H}), 1.31(\mathrm{~s}, 3 \mathrm{H}) ;{ }^{13} \mathrm{C} \mathrm{NMR}\left(\mathrm{CDCl}_{3}\right.$, $67.5 \mathrm{MHz}) \delta 165.2,132.9,130.7,130.6,130.5,128.6,118.1,109.6,108.8,82.7,81.4$, 79.6, 75.9, 72.9, 38.8, 27.3, $26.8(2 \mathrm{C}), 26.7,18.5$; HRMS (CI) calcd for $\left[\mathrm{C}_{20} \mathrm{H}_{30} \mathrm{O}_{6}+\mathrm{Na}\right]^{+}$: 389.1935, Found: 389.1941.

(6R)-5,6-dihydro-6-((E)-2'-((4'R,5'S)-2',2'-dimethyl-5'-((4"R,5”S)-2”,2”,5”trimethyl-1",3"-dioxolan-4"-yl)-1',3'-dioxolan-4'-yl)pyran-2-one (19a). 


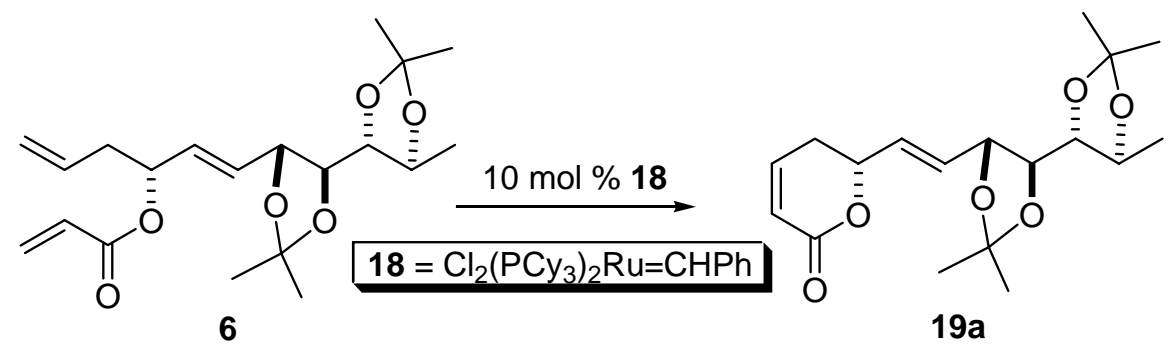

To a solution of triene $6(80 \mathrm{mg}, 0.22 \mathrm{mmol})$ in $11 \mathrm{~mL}$ of $\mathrm{CH}_{2} \mathrm{Cl}_{2}$ was added Grubbs catalyst (18 mg, $10 \% \mathrm{mmol}$ ) in $11 \mathrm{~mL} \mathrm{CH}_{2} \mathrm{Cl}_{2}$. The reaction was heated at reflux for $2 \mathrm{~h}$. Solvent was removed under reduced pressure and the residue was purifird by flash chromatography on silica gel (7:3 (v/v) hexane/EtOAc) provided lactone 19a $(61 \mathrm{mg}$, $82 \%$ yield) as a colorless oil. $\mathrm{R}_{f}=0.14\left(7: 3(\mathrm{v} / \mathrm{v})\right.$ hexane/EtOAc); IR (neat, $\left.\mathrm{cm}^{-1}\right) 2983$, 1742; $[\alpha]_{\mathrm{D}}^{25}+65.4\left(\mathrm{c} 1, \mathrm{CHCl}_{3}\right) ;{ }^{1} \mathrm{H} \mathrm{NMR}\left(\mathrm{CDCl}_{3}, 600 \mathrm{MHz}\right) \delta 6.88(\mathrm{ddd}, J=9.6,5.4$, $3.0 \mathrm{~Hz}, 1 \mathrm{H}$ ), 6.04 (ddd, $J=9.6,2.4,1.2 \mathrm{~Hz}, 1 \mathrm{H}$ ), 5.98-5.92 (m, 2H), 4.97(ddd, $J=10.8$, $4.2,4.2 \mathrm{~Hz}, 1 \mathrm{H}), 4.47$ (dd, $J=7.8,4.2 \mathrm{~Hz}, 1 \mathrm{H}), 4.02(\mathrm{dq}, J=7.8,6.0 \mathrm{~Hz}, 1 \mathrm{H}), 3.68$ (dd, $J$ $=7.8,7.2 \mathrm{~Hz}, 1 \mathrm{H}), 3.56(\mathrm{dd}, J=7.8,7.8 \mathrm{~Hz}, 1 \mathrm{H}), 2.50$ (dddd, $J=18.0,4.86,1.2,0.6 \mathrm{~Hz}$, 1H), 2.41 (dddd, $J=10.8,7.8,3.0,2.4 \mathrm{~Hz}, 1 \mathrm{H}), 1.40$ (s, 6H), 1.40 (s, 3H), 1.34 (s, 3H), $1.34(\mathrm{~d}, J=6.0 \mathrm{~Hz}, 3 \mathrm{H}) ;{ }^{13} \mathrm{C} \mathrm{NMR}\left(\mathrm{CDCl}_{3}, 125 \mathrm{MHz}\right) \delta 163.6,144.3,131.3,128.9$, 121.6, 109.8, 108.9, 82.8, 81.5, 79.4, 76.8, 76.1, 29.5, 27.3, 26.9 (2C), 26.8, 18.5; HRMS (CI) calcd for $\left[\mathrm{C}_{18} \mathrm{H}_{26} \mathrm{O}_{6}+\mathrm{Na}\right]^{+}: 361,1622$, Found: 361.1605 .

(6S)-5,6-dihydro-6-((E)-2'-((4'R,5'S)-2',2'-dimethyl-5'-((4”R,5”S)-2”,2”,5”trimethyl-1",3"-dioxolan-4"-yl)-1',3'-dioxolan-4'-yl)pyran-2-one (19b).

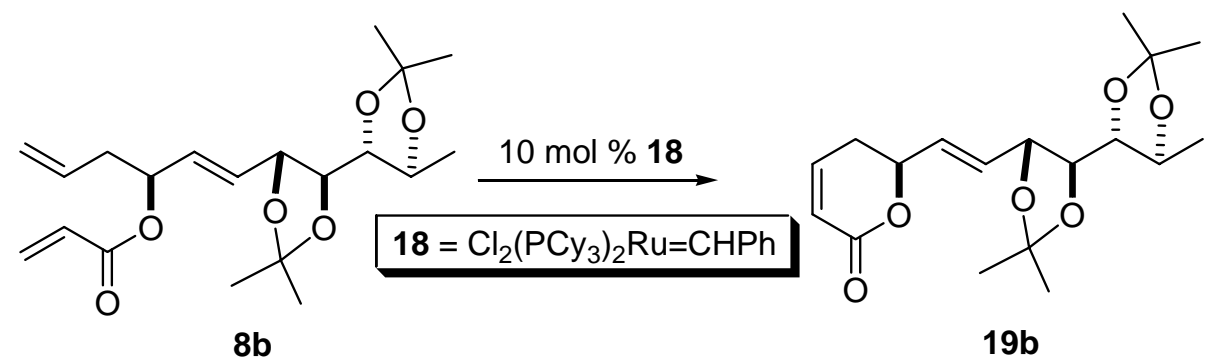

To a solution of triene $8 \mathbf{b}(57 \mathrm{mg}, 0.15 \mathrm{mmol})$ in $7.5 \mathrm{~mL}$ of $\mathrm{CH}_{2} \mathrm{Cl}_{2}$ was added Grubbs catalyst (14 mg, $10 \% \mathrm{mmol}$ ) in $7.5 \mathrm{~mL} \mathrm{CH}_{2} \mathrm{Cl}_{2}$. The reaction was heated at reflux for $3 \mathrm{~h}$. Solvent was removed under reduced pressure and the residue was purifird by flash chromatography on silica gel $(7: 3(\mathrm{v} / \mathrm{v})$ hexane/EtOAc) provided lactone $19 \mathrm{~b}(40 \mathrm{mg}$, 
$80 \%$ yield) as a colorless oil. $\mathrm{R}_{f}=0.14\left(7: 3(\mathrm{v} / \mathrm{v})\right.$ hexane/EtOAc); IR (neat, $\left.\mathrm{cm}^{-1}\right) 2983$, $1742 ;[\alpha]^{25}{ }_{\mathrm{D}}^{-4.9}\left(\mathrm{c} 1, \mathrm{CHCl}_{3}\right) ;{ }^{1} \mathrm{H} \mathrm{NMR}\left(\mathrm{CDCl}_{3}, 600 \mathrm{MHz}\right) \delta 6.88(\mathrm{ddd}, J=9.6,5.4,3.0$ $\mathrm{Hz}, 1 \mathrm{H}$ ), 6.03 (ddd, $J=10.2,1.8,1.8 \mathrm{~Hz}, 1 \mathrm{H}), 5.98-5.92$ (m, 2H), 4.97(ddd, $J=9.6,5.4$, $4.8 \mathrm{~Hz}, 1 \mathrm{H}), 4.47$ (dd, $J=7.8,4.2 \mathrm{~Hz}, 1 \mathrm{H}), 4.02$ (dq, $J=7.8,6.0 \mathrm{~Hz}, 1 \mathrm{H}), 3.66$ (dd, $J=$ 7.8, 7.2 Hz, 1H), 3.53 (dd, $J=7.8,7.2 \mathrm{~Hz}, 1 \mathrm{H}), 2.44-2.42$ (m, 2H), 1.39 (s, 6H), 1.38 (s, $3 \mathrm{H}), 1.33(\mathrm{~d}, J=6.0 \mathrm{~Hz}, 3 \mathrm{H}), 1.38(\mathrm{~s}, 3 \mathrm{H}) ;{ }^{13} \mathrm{C} \mathrm{NMR}\left(\mathrm{CDCl}_{3}, 150 \mathrm{MHz}\right) \delta 163.7,144.5$, $131.5,128.2,121.6,109.8,108.9,82.9,81.6,79.4,77.1,76.3,29.6,27.3,26.9,26.8(2 \mathrm{C})$, 18.5; HRMS (CI) calcd for $\left[\mathrm{C}_{18} \mathrm{H}_{26} \mathrm{O}_{6}+\mathrm{Na}\right]^{+}: 361.1622$, Found: 361.1605 .

\section{0-epi-anamarine (7).}

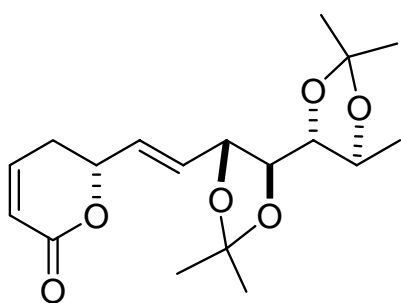

$19 \mathbf{a}$

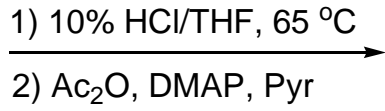

2) $\mathrm{Ac}_{2} \mathrm{O}, \mathrm{DMAP}, \mathrm{Pyr}$

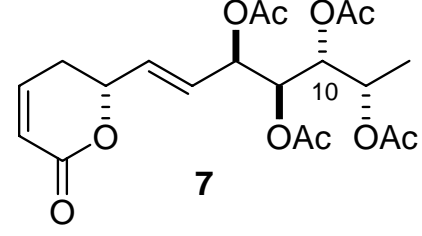

10-epi-anamarine

A solution of $10 \%$ aqueous $\mathrm{HCl}$ and $\mathrm{THF}(1: 1,1.4 \mathrm{ml})$ was added to a flask containing acetonide 19a $(27 \mathrm{mg}, 0.08 \mathrm{mmol})$. The mixture was hearted at $65{ }^{\circ} \mathrm{C}$ for $20 \mathrm{~min}$ and the solvent was removed at reduced pressure. The residue was dissolved in $1.0 \mathrm{ml}$ of pyridine, and then $\mathrm{Ac}_{2} \mathrm{O}(76 \mu \mathrm{l}, 1.6 \mathrm{mmol})$ and cat. amout DMAP were added. In $24 \mathrm{~h}$, solid $\mathrm{NaHCO}_{3}$ was added, diluted with EtOAc, and then filtered through a pad of celite. The solvent was removed under reduced pressure and the residue was purified by flash chromatography on silica gel (50:50 (v/v) hexane/EtOAc) provided 10-epi-anamarine 7 (30 mg, 86\% yield) as a white solid. $\mathrm{R}_{f}=0.15\left(1: 1(\mathrm{v} / \mathrm{v})\right.$ hexane/EtOAc); IR (neat, $\mathrm{cm}^{-1}$ ) 2925, 1745; $[\alpha]^{25}+29.8\left(c\right.$ 0.5, $\left.\mathrm{CHCl}_{3}\right) ;{ }^{1} \mathrm{H}$ NMR $\left(\mathrm{CDCl}_{3}, 600 \mathrm{MHz}\right) \delta 6.85$ (ddd, $J=$ 9.6, 6.0, 3.0 Hz, 1H), 6.03 (ddd, $J=9.6,1.2,1.2 \mathrm{~Hz}, 1 \mathrm{H}), 5.81$ (ddd, $J=15.6,5.4,1.8 \mathrm{~Hz}$, 1H), 5.68 (ddd, $J=15.6,5.4,1.2 \mathrm{~Hz}, 1 \mathrm{H}), 5.51$ (dd, $J=5.4,1.2 \mathrm{~Hz}, 1 \mathrm{H}), 5.28$ (dd, $J=$ 10.2, $2.4 \mathrm{~Hz}, 1 \mathrm{H}), 5.22(\mathrm{dd}, J=10.2,1.8 \mathrm{~Hz}, 1 \mathrm{H}), 5.10$ (dq, $J=2.4,6.6 \mathrm{~Hz}, 1 \mathrm{H}), 4.93$ (dq, $J=4.8,4.8 \mathrm{~Hz}, 1 \mathrm{H}), 2.41$ (dddd, $J=18.0,6.0,4.2,1.2 \mathrm{~Hz}, 1 \mathrm{H}), 2.34$ (dddd, $J=18.0$, 10.8, 3.0, 2.4 Hz, 1H), 2.13 (s, 3H), 2.11 (s, 3H), 2.05 (s, 3H), 2.04 (s, 3H), 1.13 (d, J= $6.6 \mathrm{~Hz}, 3 \mathrm{H}) ;{ }^{13} \mathrm{C} \mathrm{NMR}\left(\mathrm{CDCl}_{3}, 125 \mathrm{MHz}\right): \delta 170.3,170.0,169.9,169.8,163.2,144.2$, 
130.2, 127.4, 121.6, 76.3, 70.5, 69.9, 69.5, 67.1, 29.7, 21.0, 20.8, 20.6, 20.6, 16.2; HRMS (CI) calcd for $\left[\mathrm{C}_{20} \mathrm{H}_{26} \mathrm{O}_{10}+\mathrm{Na}\right]^{+}: 449.1419$, Found: 449.1403.

\section{5,10-epi,epi-anamarine (20).}

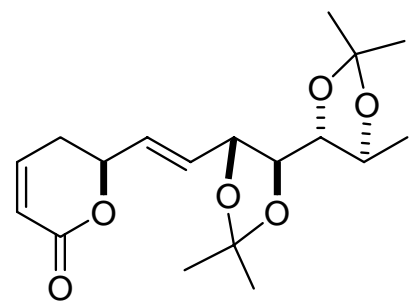

$19 b$ $\underset{\text { 1) } 10 \% \mathrm{HCl} / \mathrm{THF}, 65^{\circ} \mathrm{C}}{\longrightarrow}$

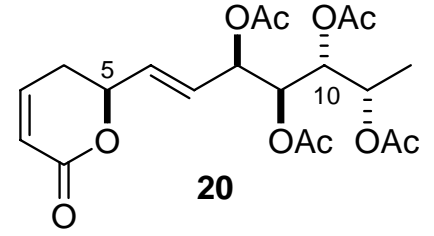

5,10-epi,epi-anamarine

A solution of $10 \%$ aqueous $\mathrm{HCl}$ and $\mathrm{THF}(1: 1,1.4 \mathrm{ml})$ was added to a flask containing acetonide 9 (44 mg, $0.13 \mathrm{mmol})$. The mixture was hearted at $65{ }^{\circ} \mathrm{C}$ for $20 \mathrm{~min}$ and the solvent was removed at reduced pressure. The residue was dissolved in $1.0 \mathrm{ml}$ of pyridine, and then $\mathrm{Ac}_{2} \mathrm{O}(124 \mu \mathrm{l}, 2.6 \mathrm{mmol})$ and cat. amout DMAP were added. In $24 \mathrm{~h}$, solid $\mathrm{NaHCO}_{3}$ was added, diluted with EtOAc and filtered through a pad of celite. The solvent was removed under reduced pressure and the residue was purified by flash chromatography on silica gel $(50: 50(\mathrm{v} / \mathrm{v})$ hexane/EtOAc) provided 5,10-epi,epianamarine 20 (47 mg, 80\% yield) as a white solid. $\mathrm{R}_{f}=0.15(1: 1(\mathrm{v} / \mathrm{v})$ hexane/EtOAc); IR (neat, $\left.\mathrm{cm}^{-1}\right)$ 2989, 1682; $[\alpha]^{25}{ }_{\mathrm{D}}^{-4} .2\left(c\right.$ 1, $\left.\mathrm{CHCl}_{3}\right) ;{ }^{1} \mathrm{H}$ NMR $\left(\mathrm{CDCl}_{3}, 600 \mathrm{MHz}\right) \delta 6.83$ (ddd, $J=9.0,5.4,3.0 \mathrm{~Hz}, 1 \mathrm{H}$ ), 6.02 (ddd, $J=10.2,1.2,1.2 \mathrm{~Hz}, 1 \mathrm{H}), 5.77$ (ddd, $J=15.6$, 6.0, $1.2 \mathrm{~Hz}, 1 \mathrm{H}), 5.68$ (ddd, $J=15.6,4.8,1.2 \mathrm{~Hz}, 1 \mathrm{H}), 5.49$ (dd, $J=3.6,1.2 \mathrm{~Hz}, 1 \mathrm{H})$, $5.30(\mathrm{dd}, J=10.2,2.4 \mathrm{~Hz}, 1 \mathrm{H}), 5.21(\mathrm{dd}, J=10.2,2.4 \mathrm{~Hz}, 1 \mathrm{H}), 5.09(\mathrm{qd}, J=6.6,1.8 \mathrm{~Hz}$, 1H), 4.87(dq, $J=4.8,4.8 \mathrm{~Hz}, 1 \mathrm{H}), 2.41$ (dddd, $J=18.6,5.4,4.8,1.2 \mathrm{~Hz}, 1 \mathrm{H}), 2.35$ (dddd, $J=18.6,10.2,3.0,2.4 \mathrm{~Hz}, 1 \mathrm{H}), 2.12(\mathrm{~s}, 3 \mathrm{H}), 2.10(\mathrm{~s}, 3 \mathrm{H}), 2.06(\mathrm{~s}, 3 \mathrm{H}), 2.03(\mathrm{~s}, 3 \mathrm{H}), 1.13$ $(\mathrm{d}, J=6.6 \mathrm{~Hz}, 3 \mathrm{H}) ;{ }^{13} \mathrm{C} \mathrm{NMR}\left(\mathrm{CDCl}_{3}, 125 \mathrm{MHz}\right) \delta 170.3,169.9,169.9,169.7,163.3$, 144.1, 130.0, 127.8, 121.6, 70.5, 69.9, 69.4, 67.0, 67.0, 29.6, 29.4, 20.9, 20.8, 20.6, 16.1; HRMS (CI) calcd for $\left[\mathrm{C}_{20} \mathrm{H}_{26} \mathrm{O}_{10}+\mathrm{Na}\right]^{+}:$449.1419, Found: 449.1423. 